\title{
High-pressure study of microcrystalline tungsten trioxide phase transitions by Raman spectroscopy
}

\author{
M. Boulova ${ }^{1,2}$, G. Lucazeau', T. Pagnier ${ }^{1}$ and A. Gaskov ${ }^{2}$ \\ ${ }^{1}$ LEPMI, CNRS INPG, 1130 rue de la Piscine, BP. 75, 38402 Saint-Martin-d'Hères cedex, \\ France \\ ${ }^{2}$ Chemistry Department, Moscow State University, Moscow 119899, Russia
}

\begin{abstract}
A high-pressure Raman study of microcrystalline tungsten oxide was performed in the $0.1 \mathrm{MPa}-31 \mathrm{GPa}$ pressure range under isostatic and non-isostatic conditions. The phase transitions, which take place below $0.1 \mathrm{GPa}$ and at $22 \mathrm{GPa}$ are first order, while two further structural changes, observed at about 3 and $10 \mathrm{GPa}$, are diffuse. Surprisingly, the non-isostatic conditions do not induce inhomogeneous band broadenings and do not modify the transitions sequence observed in isostatic conditions. Compressibility of the different phases is estimated from spectral data.
\end{abstract}

\section{INTRODUCTION.}

$\mathrm{WO}_{3}$ is known to be complex in terms of structure and phase transitions. At room temperature the coexistence of monoclinic $\gamma\left(P 2_{1} / n, Z=8\right)$ and triclinic $\delta(P \overline{1}, Z=8)$ [1-3] phases was reported. Temperature changes of $\mathrm{WO}_{3}$ structure under atmospheric pressure can be presented as follows: triclinic $(\mathrm{Z}=8)$ $\stackrel{233 \mathrm{~K}}{\longrightarrow}$ monoclinic $\varepsilon(\mathrm{Pc}) \stackrel{246 \mathrm{~K}}{\longrightarrow}$ triclinic $\delta \stackrel{290 \mathrm{~K}}{\longrightarrow}$ monoclinic $\gamma \stackrel{600 \mathrm{~K}}{\longrightarrow}$ orthorhombic (Pmnb, $\mathrm{Z}=8) \stackrel{1010 \mathrm{~K}}{\longrightarrow}$ tetragonal $(\mathrm{P} 4 / \mathrm{nmm}, \mathrm{Z}=2)[2,4-6]$. The existent data on high-pressure (HP) measurements of tungsten trioxide phase transition are not so clear. $\mathrm{Xu}$ and al. [2] have found that the triclinic form of $\mathrm{WO}_{3}$ transits towards a $\mathrm{HP}$ phase at $0.57 \mathrm{GPa}$. Salje and Hoppmann [7] revealed that initial monoclinic $\gamma$-phase of $\mathrm{WO}_{3}$ crystal partially transforms firstly to triclinic $\delta$-phase at $0.1 \mathrm{GPa}$, and after to Pc phase at $0.155 \mathrm{GPa}$. Souza-Filho et al. [8] have analyzed the Raman spectra of microcrystallized $\mathrm{WO}_{3}$ in the $0.01-5 \mathrm{GPa}$ pressure range. They observed the successive disappearance of monoclinic $\gamma$ - and triclinic $\delta$ - phases at $1.4 \mathrm{GPa}$ and development of HP phase until to $1.01 \mathrm{GPa}$ without real arguments to decide about its structure

The aim of this investigation was to follow the effect of hydrostatic and non-hydrostatic compressive stresses on the structure of tungsten oxide more precisely inside a wide pressure interval.

\section{EXPERIMENTAL PROCEDURE}

Powder of tungsten oxide (99.995\% pure) was obtained from Aldrich. X-ray diffraction diagram reveals that it is the mixture of monoclinic $(\gamma) \mathrm{WO}_{3}$ phase and of triclinic $(\delta) \mathrm{WO}_{3}$ phase in the approximate ratio 90:10.

Raman spectra were obtained using an XY Dilor multi-channel spectrometer equipped with a CCD detector. The $514.53 \mathrm{~nm}$ line from an Argon ion laser was used as the excitation source. Incident powers of about $5 \mathrm{~mW}$ on the sample were used. A diamond anvil cell (DAC) was used for HP experiments. For obtaining isostatic pressure a 4:1 ethanol-methanol mixture was used as pressure transmitting medium. Non-isostatic pressure investigations consisted in studying the powder without transmitting medium. 


\section{RESULTS}

Raman spectra obtained under isostatic HP conditions at room temperature from atmospheric to $31 \mathrm{GPa}$ are shown in fig. 1. Our DAC is not adapted to very low-pressure values (in the $10 \mathrm{MPa}-0.2 \mathrm{GPa}$ region).

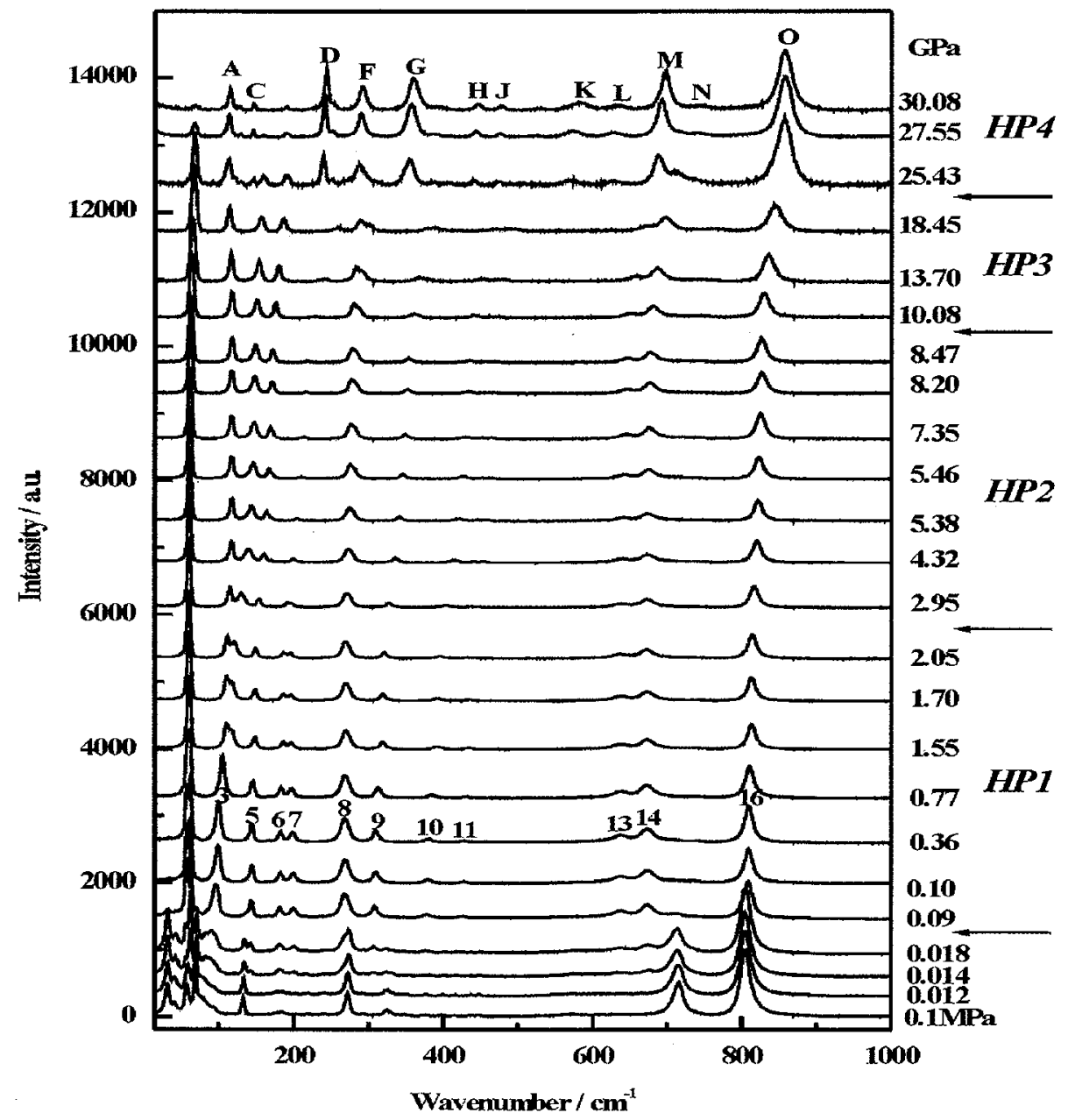

Fig. 1. Raman spectra versus hydrostatic pressure from atmospheric to $31 \mathrm{GPa}$ (Raman bands symbols marked by numbers and letters).

Therefore the pressure values for this interval were estimated from the values of primary external pressure. The pressure dependence of all Raman bands frequency is plotted in Fig.2. This dependence is rather linear in the HP4 pressure domain separated by dotted lines at $22 \mathrm{GPa}$. On the contrary the pressure dependence of low frequency modes is strongly non linear between 0.01 and $5 \mathrm{GPa}$. The $\partial v / \partial P$ quantities have been derived for each mode in each phase and reported in table 1 . Some of these derivatives are negative as for some high frequency modes and strongly positive for some low-frequency mode, such as that at about $100 \mathrm{~cm}^{-1}$ (see fig.2). The region of strong changes of $\partial v / \partial P$ around $3 \mathrm{GPa}$ has been considered as possibly due to a phase change. 
The results of HP study without transmitting medium are quite close to the previous ones and are not reproduced here.
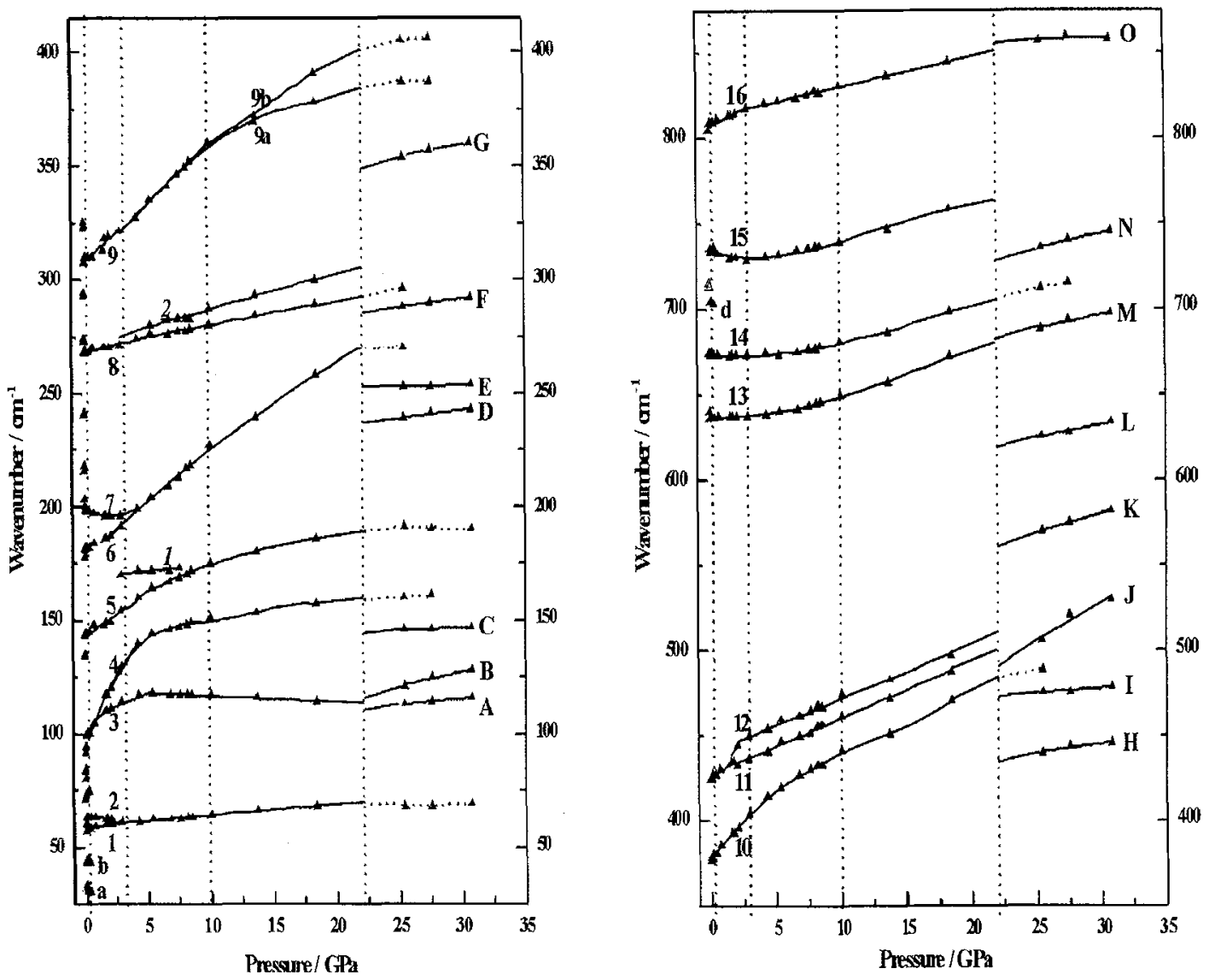

Fig.2. Pressure dependence of Raman frequency for all Raman bands.

\section{DISCUSSION}

Between $10 \mathrm{MPa}$ - $31 \mathrm{GPa}$ two first order phase transitions at about 0.1 and $22 \mathrm{GPa}$ respectively, and probably two second order transitions at about 3 and $10 \mathrm{GPa}$ are evidenced. In the initial spectrum of monoclinic and triclinic phases mixture the number of Raman bands is about 30 . After the first phase transition to $H P I$ phase this number decreases and then keeps almost the same in all phases: sixteen for $H P 1$ phase, seventeen for HP2 and HP3 and fifteen for HP4. This means that $\mathrm{WO}_{3}$ does not undergo strong symmetry and multiplicity cell changes through this investigated pressure range. 
Table 1. Raman wavenumber of different phases.

\begin{tabular}{|c|c|c|c|c|c|c|c|c|c|}
\hline \multirow{2}{*}{$\begin{array}{l}\text { Band } \\
\text { symbol }\end{array}$} & \multicolumn{4}{|c|}{$\frac{0.1 G P a-3 G P a}{\mathbf{H P 1}}$} & \multicolumn{2}{|c|}{$\begin{array}{c}3-\sim 22 G P a \\
\mathbf{H P 2}, \mathbf{H P 3}\end{array}$} & \multicolumn{2}{|c|}{$\begin{array}{c}\sim 22 G P a \rightarrow \\
\mathbf{H P 3}\end{array}$} & \multirow{2}{*}{$\begin{array}{l}\text { Band } \\
\text { symbol }\end{array}$} \\
\hline & $\begin{array}{c}v_{0.36 \mathrm{GPa}} \\
\left(\mathrm{cm}^{-1}\right)\end{array}$ & $\left(\frac{\partial \boldsymbol{v}}{\partial \mathbf{P}}\right)_{r}$ & $\begin{array}{l}v_{2.05 G \mathrm{~Pa}} \\
\left(\mathrm{~cm}^{-1}\right)\end{array}$ & $\left(\frac{\partial v}{\partial \mathrm{P}}\right)_{T}$ & $\begin{array}{l}v_{13.7 \mathrm{GPa}} \\
\left(\mathrm{cm}^{-1}\right)\end{array}$ & $\left(\frac{\partial v}{\partial \mathrm{P}}\right)_{T}$ & $\begin{array}{l}v_{30,1 \mathrm{GPa}} \\
\left(\mathrm{cm}^{-1}\right)\end{array}$ & $\left(\frac{\partial v}{\partial \mathrm{P}}\right)_{T}$ & \\
\hline a & $30.5^{w}$ & & & & & & & & \\
\hline$b$ & $44.2^{w}$ & & & & & & & & \\
\hline 1 & $58.0^{\mathrm{s}}$ & +1.5 & $60.1^{s}$ & +0.4 & $65.7^{\mathrm{s}}$ & +0.5 & & & \\
\hline 2 & $62.7^{\mathrm{s}}$ & -0.7 & $62.1^{s}$ & -0.8 & & & & & \\
\hline 3 & $99.5^{5}$ & +9.0 & $111.7^{\mathrm{m}}$ & +2.9 & $116.2^{\mathrm{m}}$ & -0.3 & & & \\
\hline 4 & & & $121.0^{\mathrm{m}}$ & +9.7 & $153.4^{\mathrm{m}}$ & +0.9 & $115.6^{\mathrm{m}}$ & +0.6 & A \\
\hline & & & & & & & $\begin{array}{l}128.5^{\mathrm{w}} \\
147.1^{\mathrm{w}}\end{array}$ & $\begin{array}{l}+1.3 \\
+0.2\end{array}$ & $\begin{array}{l}\mathrm{B} \\
\mathrm{C}\end{array}$ \\
\hline 5 & $143.8^{\mathrm{m}}$ & +7.1 & $149.5^{\mathrm{m}}$ & +2.7 & $179.9^{\mathrm{m}}$ & +1.6 & & & \\
\hline 6 & $182.0^{\mathrm{m}}$ & +3.7 & $186.7^{\mathrm{m}}$ & +3.1 & $241.2^{\mathrm{w}}$ & +4.1 & & & \\
\hline 7 & $198.2^{\mathrm{m}}$ & -3.5 & $195.6^{\mathrm{m}}$ & -0.4 & & & & & \\
\hline & & & & & & & $\begin{array}{l}243.2^{\mathrm{m}} \\
253.8^{\mathrm{w}}\end{array}$ & $\begin{array}{l}+0.7 \\
+0.2\end{array}$ & $\begin{array}{l}\mathrm{D} \\
\mathrm{E}\end{array}$ \\
\hline 8 & $268.4^{\mathrm{m}}$ & +2.1 & $270.0^{\mathrm{m}}$ & +0.4 & $283.8^{\mathrm{m}}$ & +1.1 & $292.2^{\mathrm{m}}$ & +0.7 & $F$ \\
\hline$\frac{2}{9}$ & $309.6^{\mathrm{m}}$ & +5.5 & $320.9^{\mathrm{m}}$ & +6.2 & $292.4^{w}$ & +1.6 & & & \\
\hline $9 a$ & & & & & $369.0^{\mathrm{W}}$ & +2.5 & & & \\
\hline $9 b$ & & & & & $372.0^{\mathrm{w}}$ & +3.8 & 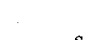 & & \\
\hline 10 & $379.6^{w}$ & +9.2 & $395.0^{\mathrm{w}}$ & +7.9 & $451.8^{\mathrm{w}}$ & +3.7 & $360.1^{s}$ & +1.1 & G \\
\hline & & & & & & & $\begin{array}{l}445.9^{w} \\
477.4^{w}\end{array}$ & $\begin{array}{l}+1.1 \\
+0.6\end{array}$ & $\begin{array}{l}\mathrm{H} \\
\mathrm{I}\end{array}$ \\
\hline 11 & $426.2^{w}$ & +5.5 & 431.0 & +6.3 & $471.3^{w}$ & +3.2 & & & \\
\hline 12 & & & 444.0 & +4.8 & $482.0^{\mathrm{w}}$ & +3.0 & & & \\
\hline & & & & & & & $528.2^{\mathrm{w}}$ & +4.3 & $\mathrm{~J}$ \\
\hline & & & & & & & $581.9^{w}$ & +2.3 & K \\
\hline & & & & & & & $633.2^{\mathrm{w}}$ & +1.5 & $\mathrm{~L}$ \\
\hline 13 & $636.4^{\mathrm{m}}$ & +1.9 & $636.4^{\mathrm{m}}$ & 0.0 & $657.0^{w}$ & +2.6 & & & \\
\hline 14 & $673.1^{\mathrm{II}}$ & 0.0 & $672.8^{\mathrm{m}}$ & +0.1 & $686.4^{\mathrm{m}}$ & +1.9 & & & \\
\hline d & $703.8^{\mathrm{w}}$ & -4.0 & & & & & $697.0^{s}$ & +1.6 & M \\
\hline & & & & & & & $745.0^{\mathrm{w}}$ & +1.8 & $\mathrm{~N}$ \\
\hline 15 & $734.5^{\mathrm{w}}$ & -4.1 & $730.3^{w}$ & -0.3 & $745.5^{\mathrm{w}}$ & +2.0 & & & \\
\hline 16 & $809.4^{\mathrm{s}}$ & +3.7 & $814.5^{\mathrm{s}}$ & +2.7 & $835.6^{\mathrm{s}}$ & +1.8 & & & \\
\hline & & & & & & & $858.2^{\mathrm{s}}$ & +0.3 & $\mathrm{O}$ \\
\hline
\end{tabular}

a) Immediately after placing the sample into the cell, the spectrum changes. At $0.1 \mathrm{GPa}$ the transformation into HP1 phase is complete. The same phase transition was observed by Salje [7], Souza Filho [8] and $\mathrm{Xu}$ [2], but at higher-pressure values: $0.155 \mathrm{GPa}$ and $1.01 \mathrm{GPa}$ respectively. According to the above literature the HPl phase is observed up to $5 \mathrm{GPa}$. However, our data give a strong evidence that a second order transition takes place at about $3 \mathrm{GPa}$ and that the existence of HP1 is smaller than that reported previously. Number of Raman modes stays the same as for initial phase although the $Z$ decreases from 8 to 4 according to data of [2].

Calculation of compressibility value using standard Hazen's model gives a bulk modulus of 625 $\mathrm{GPa}$ for the HP1 phase. Such a value, even much larger than for diamond (about $400 \mathrm{GPa}$ ), is unrealistic. Such a discrepancy between calculated and observed compressibilities is not met for usual perovskites, likely because the large dodecahedral cavity is occupied by a cation, electrons of which via oxygen-electron repulsions, contribute to decrease the compressibility. For obtaining the 
right order of magnitude of bulk modulus, we propose an extension of Hazen's model consisting in the introduction of an adjustable hole transfer in the unoccupied dodecahedral.

Using spectral data (derivatives $d v / d p$, listed in table 1) we obtained the bulk modulus of 24.3 GPa for the HP1 phase. This value is of the same order of magnitude as that reported by Xu (44 $\mathrm{GPa}$ [2].

b) At about $3 \mathrm{GPa}$ a pronounced change in the slopes of the $v=f(P)$ curves is observed and some new features appear in the spectrum(bands $\underline{1}, \underline{2}$ in fig.2). These facts indicate that a new phase, noted $H P 2$, is probably formed. The absence of discontinuity in the wavenumbers evolutions suggests that it is a second order phase transition. The calculated bulk modulus value for this phase is $515 \mathrm{GPa}$. Moreover, the splitting of the line 9 into components $9 \mathrm{a}$ and $9 \mathrm{~b}$ could suggest the existence of an additional transition near $10 \mathrm{GPa}$, which has to be confirmed later.

c) Once more, above $10 \mathrm{GPa}$, subtle changes can be noticed: the band $\underline{\underline{l}}$ merges into band 5 , and band 9 splits into two components $9 \mathrm{a}$ and $9 \mathrm{~b}$, suggesting that a structural change (to the HP3 phase) might have taken place.

d) At high pressure, between 18 and $25 \mathrm{GPa}$, the full change of Raman spectrum was observed. With the disappearance of $H P 3$ bands, strong new features are observed, indicating that the HP3 phase transforms into a new HP4 phase and that the transition is of first order. For this phase transition one can notice the survival of some HP3 bands above the transition pressure. It is interesting to note that the $v=f(P)$ curves of surviving HP3 lines (doted lines in fig. 2) are nearly horizontal. This can be related with the fact that the local pressure remains constant during the complete transformation of this phase. The existence of a pressure gradient at such high pressures can explain that some parts of the sample are already in the $H P 4$ phase while others are still under transformation, it is for these parts that the local pressure is fixed. This remark can help to precise the pressure value for the transition by the extrapolating $v(\mathrm{P})$ both in HP3 and HP4 sides, for e.g. mode number 6 (fig.2) the horizontal dotted line intersects the corresponding continuous curve at about $22 \mathrm{GPa}$.

When the pressure inside the cell is released, the spectrum taken at $0.1 \mathrm{MPa}$ corresponds to $H P I$ phase. After one day, the transformation of this spectrum into the spectrum of triclinic $\delta$ phase is observed.

The high-pressure study of tungsten oxide without transmitting medium gives unexpectedly quite similar results. In particular the band's full-width at half-maximum (FWHM) in these non isostatic conditions remain close to those measured in isostatic conditions and this fact is completely unusual as if tungsten oxide was extremely isotropic although its different crystalline forms suggest the contrary.

\section{CONCLUSION}

A Raman high-pressure study has been performed on pure tungsten oxide up to $31 \mathrm{GPa}$. Three clear phase transitions were found in this region of pressure: at about $0.01 \mathrm{GPa}$, near $3 \mathrm{GPa}$ and between 18 and 25 $\mathrm{GPa}$. We are certain that the transition about $3 \mathrm{GPa}$ is of the second order and the highest one - is of the first order. Also suggestion about the one more transition of second order at about $10 \mathrm{GPa}$ is proposed. The first low-pressure transition correlates with reported one in the literature, but it happens at lower pressure value in our case. In the high-pressure domain tungsten oxide transforms towards the more high symmetric structure, but not yet cubic. 


\section{References}

[1] Salje E. and Viswanathan K., Acta Cryst. A 31 (1975) 356-359.

[2] Xu Y., Carlson S., and Norrestam R., J. Solid State Chem. 132 (1997) 123-130.

[3] Souza-Filho A.G., Freire V.N., Sasaki J.M., Filho J.M., Juliao J.F., and Gomes U.U., J. Raman Spectroscopy 31 (2000) 451-454.

[4] Daniel M.F., Desbat B., Lassegues J.C., Gerand B., and Figlarz M., J. Solid State Chem. 67 (1987) 235-247.

[5] Cazzanelli E., Vinegoni C., Mariotto G., Kuzmin A., and Purans J., J.Solid State Chem. 143 (1999) 2432.

[6] Diehl R. and Brandt G., Acta Cryst. B34 (1978) 1105-1111.

[7] Salje E. and Hoppmann G., High Temp. High Press. 12 (1980) 213-216.

[8] Souza-Filho A.G., Freire V.N., Pilla O., Ayala A.P., Filho J.M., Melo F.E.A., Freire V.N., and Lemos V., Phys. Rev. B 62 (2000) 3699-3703. 